\title{
Association Between Adherence to Maintenance Medication in Patients with COPD and Acute Exacerbation Occurrence and Cost in China: A Retrospective Cohort Database Study
}

This article was published in the following Dove Press journa

International Journal of Chronic Obstructive Pulmonary Disease

\author{
Rongchang Chen' \\ Yue $\mathrm{Gao}^{2}$ \\ He Wang ${ }^{3}$ \\ Hongyan Shang ${ }^{3}$ \\ Jianwei Xuan ${ }^{4}$
}

'State Key Laboratory of Respiratory Disease, National Clinical Research

Center, Guangzhou Institute of Respiratory Health, First Affiliated Hospital of Guangzhou Medical University, Guangzhou, People's Republic of China; ${ }^{2}$ Health Economics, Shanghai Centennial Scientific Co. Ltd., Shanghai, People's Republic of China; ${ }^{3}$ Medical Affairs, AstraZeneca China, Shanghai, People's Republic of China; ${ }^{4} \mathrm{Health}$ Economics Research Institute, Sun YatSen University, Guangzhou, People's Republic of China
Correspondence: Rongchang Chen State Key Laboratory of Respiratory Disease, National Clinical Research Center, Guangzhou Institute of Respiratory Disease, First Affiliated Hospital of Guangzhou Medical University, Guangzhou, People's Republic of China

Tel +862083062870

Email chenrc@vip.l63.com
Background: This study aimed to evaluate the association between adherence to maintenance medication (ie, inhaled bronchodilators, inhaled corticosteroid/long-acting beta-2 agonist [ICS/LABA] combinations, and oral therapy) and acute exacerbation of chronic obstructive pulmonary disease (AECOPD) and related costs among patients with chronic obstructive pulmonary disease (COPD) in China.

Patients and Methods: Claims data from the hospitals of a metropolitan city in south China between January 2014 and December 2016 were obtained. Patients with COPD with $\geq 2$ maintenance medication claims during 1 year were included. Adherence was measured by the proportion of days covered (PDC). The interaction of medication class $\times$ adherence was considered when building models.

Results: A total of 11,708 patients met the inclusion criteria, of whom $10.8 \%$ were highly adherent ( $P D C \geq 0.8$ ). There were significant interaction effects of drug category on hospitalized AECOPD risk $(\mathrm{P} \leq 0.001)$, hospitalized AECOPD rate $(\mathrm{P}<0.001)$, and 1-year hospitalized AECOPD treatment costs $(\mathrm{P}=0.012)$. There was a relationship between high adherence and outcomes for ICS/LABA combinations $(n=3,419)$, ie, relative risk of hospitalized AECOPD was reduced by $34.8 \%$ (adjusted odds ratio $=0.65 ; 95 \%$ confidence interval (CI): 0.54-0.79; $\mathrm{P}<0.001)$ while the frequency of hospitalized AECOPD per patient-year was reduced by $24.4 \%$ (adjusted rate ratio $=0.76 ; 95 \% \mathrm{CI}: 0.65$ to $0.87 ; \mathrm{P}<0.001$ ). Mean 1 -year per-patient hospitalized AECOPD costs were reduced by $37.8 \%$ (mean difference $=-848$ USD; 95\% CI: $-1435-262$ USD; $\mathrm{P}<0.001)$. Patients taking oral mucolytics and having high adherence had worse AECOPD outcomes than patients with poor adherence.

Conclusion: High adherence to ICS/LABA maintenance therapy was associated with reduced hospitalized AECOPD rates and costs in Chinese patients with COPD.

Keywords: chronic obstructive pulmonary disease, adherence, acute exacerbation of chronic obstructive pulmonary disease, cost, ICS/LABA

\section{Introduction}

Chronic obstructive pulmonary disease (COPD) is a progressive and non-reversible airflow limitation disease and is a leading cause of morbidity and mortality worldwide. ${ }^{1}$ Estimates from the global burden of disease 2016 study show that COPD was the fourth, sixth, and seventh leading cause of years of life lost (YLLs) in countries with middle-income, high-income, and high-middle income, respectively. In China, COPD 
ranks as the third and fourth leading cause of death in rural and urban areas, respectively. ${ }^{2}$

A study of the disease burden of COPD found that the annual direct medical expense for Chinese urban patients in 2006 was 1970 USD, and the estimated indirect cost was 264 USD per patient. ${ }^{3}$ Total medical expense for each COPD patient accounted for $40 \%$ of the average family income. ${ }^{4}$ Evidence showed that the cost of COPD was strongly correlated with the severity of disease, hospitalization being a major contributor to medical expenses. ${ }^{5}$

Despite its high prevalence and economic burden, and contrary to the guidelines of the Global Initiative for Chronic Obstructive Lung Disease (GOLD), inappropriate management is common in COPD and include the overuse of mucolytics. ${ }^{3,6,7}$ Indeed, in real-world settings, compliance with treatment guidelines is much lower among patients with COPD compared with that in clinical trials. ${ }^{8-10}$ A number of studies in the USA also showed that poor adherence to maintenance medication is negatively correlated with healthcare resource utilization and costs. ${ }^{11-13}$ Nevertheless, no study has examined the clinical and economic consequences of poor adherence to maintenance medication in COPD in a real-world setting in China. The purpose of this study, therefore, was to evaluate the association between adherence to maintenance medication and the occurrence of acute exacerbation of COPD (AECOPD) and associated cost among patients with COPD in China.

\section{Patients and Methods}

\section{Patients}

The study was a retrospective cohort study. Data of claims made between January 1, 2014, and December 31, 2016, from a metropolitan city in south China were obtained from the Medical Claims Database, covering all patients insured by urban employee/resident medical insurance. The registered resident population is over 13 million, and more than 10 million of them are insured. The index date was the date of the first prescription fill of COPD maintenance medication in 2015. The patients were followed for 1 year before and 1 year after the index date. Information extracted included demographics, diagnosis, treatment, medication, and costs. Because only de-identified records were used in the analysis, ethical approval and informed consent were not required, as per the local medical research policy when using electronic health data.
The inclusion criteria were 1) diagnosed with COPD in 2015 (including patients with past COPD history); 2) had continuous medical insurance coverage for 1 year prior to and 1 year after the index date through December 31,2016; and 3) had $\geq 2$ COPD maintenance medication claims during follow-up. The patients were excluded if they had other chronic respiratory conditions such as lung cancer, pulmonary fibrosis, or asthma.

\section{COPD Maintenance Medication}

There were three categories of maintenance medications considered for analysis: inhaled bronchodilators alone (long-acting beta-2 agonist [LABA] and/or long-acting muscarinic antagonist [LAMA]), combination therapy with inhaled corticosteroid (ICS)/LABA, and oral therapy (theophylline and/or mucolytics). Patients were categorized into the three groups by medication category prescribed on the index date.

\section{Adherence}

Treatment adherence was based upon the calculated proportion of days covered (PDC). For the purposes of this study, we calculated PDC in terms of the number of days with COPD maintenance medication divided by the duration of therapy (DOT) with these agents (365 days). Because the database lacked medication order information, the DOT for each prescription was based on expert opinion concerning the Chinese clinical practice in this region. For oral medications, a prescription was assumed to last for 14 days, and for 30 days for inhalation therapies. PDC ranged from 0 to 1 . Patients were deemed highly adherent if their PDC was $\geq 0.8$ and poorly adherent if their PDC was $<0.8$ during follow-up. A sensitivity analysis was undertaken for PDC at a cutoff point of 0.5.

\section{Exacerbation Identification}

Moderate exacerbation was defined as requiring treatment with systemic corticosteroids and/or antibiotics in an outpatient setting. Severe exacerbation was defined as requiring hospitalization or emergency department visit.

\section{Outcomes}

The outcomes were the risk of AECOPD, AECOPD rate (frequency), and AECOPD treatment cost per patient-year. All costs were in the perspective of the health payer. The costs included the costs for drugs, examinations, tests, and physician consultation. 


\section{Statistical Analysis}

Descriptive statistics of patient baseline characteristics (ie, demographics, comorbidities), clinical characteristics including COPD severity indicators (ie, exacerbation frequency), healthcare resource utilization (ie, COPD medication usage and hospital visits), and costs were conducted in the 1-year pre-index period in both the high and poor adherence groups. The Kruskal-Wallis test for continuous variables and the chi-square test for categorical variables were used to determine if the differences between groups (high vs poor adherence) were statistically significant.

Three multivariate regression models adjusted for baseline characteristics were used to test the difference between the high and poor adherence groups for the outcomes during the 1-year follow-up. A logistic regression model was developed to evaluate the risk of hospitalized AECOPD occurrence; a negative binomial regression model was developed to evaluate the hospitalized AECOPD frequency; and a generalized linear model with gamma distribution and log-link function was developed to evaluate hospitalized AECOPD cost. Odds ratios (ORs), relative risks (RRs), and mean differences (MDs) with their respective $95 \%$ confidence intervals (CIs) were presented. A two-tailed $\mathrm{P}<0.05$ was considered to be statistically significant.

In all models above, the interaction of medication class $\times$ adherence was constructed to explore whether the influence of adherence to healthcare resource utilization/ AECOPD occurrence was modified by medication class.

A subgroup analysis was performed in patients with COPD with exacerbation history. A supplementary analysis was also performed PDC $>0.5$ to define high adherence. In the sensitivity analyses, a propensity score matching method was used to ensure that the two groups, ie, high adherence vs poor adherence to the ICS/LABA combination, were balanced. All baseline characteristics (demographics, comorbidities, exacerbation frequency, COPD medication usage, hospital visit, and costs) were included in the matching model. A greedy algorithm (nearest match with maximum caliper $=0.02$ and 0.008 ) without replacement was used to match patients with a 1:1 matching ratio.

All analyses were conducted with STATA SE 12.0 (Stata Corp, College Station, Texas, USA).

\section{Results}

A total of 11,708 eligible patients were identified from the database, of which 1261 patients (10.8\%) were highly adherent with maintenance medication (PDC $\geq 0.8$ ). Among them, 620 patients used inhaled bronchodilators $(\mathrm{n}=225$ and $\mathrm{n}=395$ in the high and poor adherence groups, respectively); 3419 patients used the ICS/LABA combination ( $\mathrm{n}=903$ and $\mathrm{n}=2516$ in the high and poor adherence groups, respectively); and 7669 patients used oral therapy ( $n=133$ and $n=7536$ in the high and poor adherence groups, respectively) (Figure 1, Supplementary Table S1).

Baseline characteristics for each medication group are described in Table 1 and Supplementary Tables S2 and S3. The high adherence group had higher AECOPD frequency, more COPD medication prescriptions, and spent more on COPD during the pre-index period.

There was a significant interaction effect of drug category for hospitalized AECOPD risk, hospitalized AECOPD rate, and hospitalized AECOPD treatment cost during the 1-year follow-up period (adjusted p-values: $<0.001,<0.001$, and 0.012 , respectively), indicating that the association between adherence and outcome varied between maintenance medication categories. Further analysis showed no protective effect of high adherence upon clinical and economic outcomes in the inhaled bronchodilator and oral medication groups.

Results of logistic regression showed that for patients with COPD using the ICS/LABA combination maintenance therapy, the relative risk of hospitalized AECOPD in the high adherence group was reduced by $34.8 \%$ compared with those who were poorly adherent (adjusted OR: 0.65 ; standard error [SE]: 0.06; 95\% CI: 0.54, 0.79; $\mathrm{p}<0.001$ ) (Table 2 and Supplementary Table S4). Interestingly, patients taking oral mucolytics and having high adherence had a higher relative risk of hospitalized COPD than patients with poor adherence (adjusted $\mathrm{OR}=2.51 ; \quad \mathrm{SE}: \quad 0.55 ; \quad 95 \% \quad \mathrm{CI}: 1.63,3.87 ; \mathrm{p}<0.001)$ (Table 2).

In addition, results of the negative binomial regression showed that compared with those who were poorly adherent, patients achieving high adherence to the ICS/LABA combination maintenance therapy had a $24.4 \%$ reduced rate (frequency) of hospitalized AECOPD per patientyear (RR: 0.76; SE: 0.06; 95\% CI: 0.65, 0.87; $<<0.001$ ) (Table 3 and Supplementary Table S4). Compared with those who were poorly adherent, patients achieving high adherence to oral mucolytics maintenance therapy had an $82 \%$ higher rate (frequency) of hospitalized AECOPD per patient-year (RR: 1.82; SE: 0.21; 95\% CI: 1.45, 2.29; $\mathrm{p}<0.001$ ) (Table 3). 


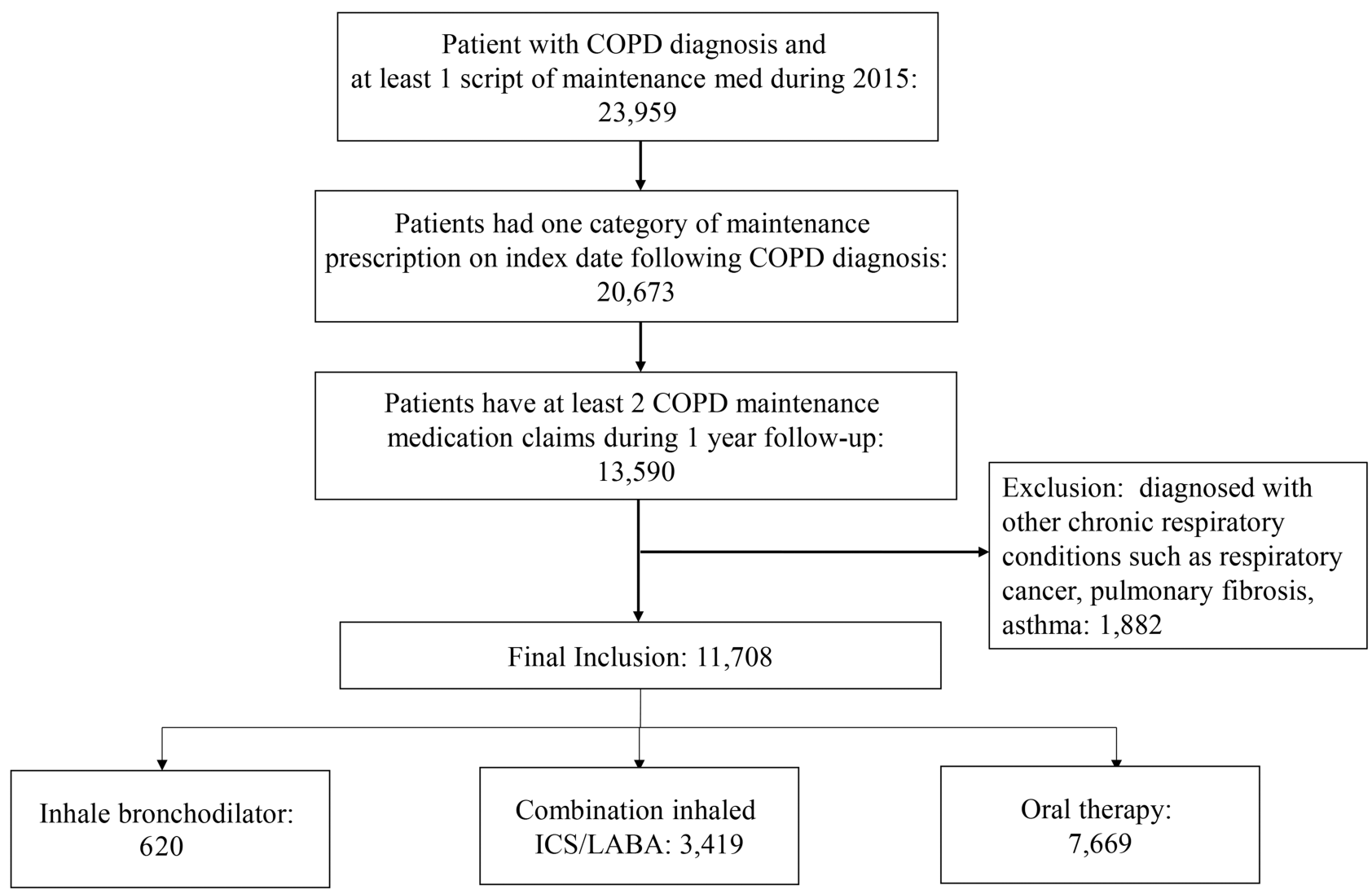

Figure I Patient flowchart.

Abbreviations: COPD, chronic obstructive pulmonary disease; ICS/LABA, inhaled corticosteroid/long-acting beta-2 agonist.

Finally, the results of the generalized linear regression showed that for patients with COPD using the ICS/LABA combination, hospitalized AECOPD costs were reduced by $37.8 \%$ if the patients were highly adherent vs those poorly adherent. Predicted mean costs were 1393 vs 2242 USD, and the adjusted mean difference (SE) was -848 USD (299) (95\% CI: $-1435,-262 ; \mathrm{p}<0.001)$ (Table 4 and Supplementary Table S4). For patients with COPD using the oral mucolytics, predicted mean costs were 5273 vs 2053 USD, and the adjusted mean difference (SE) was 3221 USD (1204) (95\% CI: 860, 5581; $\mathrm{p}<0.001)$ (Table 4).

\section{Subgroup Analysis}

Patients with COPD with exacerbation history were included in the subgroup analysis of 6011 patients, of which 800 patients were in the high adherence group, and 5211 patients were in the poor adherence group. Among patients using the ICS/LABA combination, upon controlling for baseline covariates, compared with the poor adherence group, the risk of hospitalized AECOPD occurrence for the high adherence group was decreased by $44 \%$ (adjusted OR: $0.56, \mathrm{p}<0.001$ ) while the rate (frequency) of hospitalized AECOPD per patient-year was decreased by $24 \%$ ( 0.64 vs 0.84 , adjusted rate ratio $=0.76, p=0.002)$. Patients in the high adherence group had 37.7\% lower hospitalized AECOPD costs (adjusted mean difference: $-1,029$ USD; 95\% CI: $-1,839,-219$ USD; $p=0.002$ ).

\section{Propensity Score Matching}

With propensity score matching, 734 COPD patients with high adherence to the ICS/LABA combination maintenance therapy were matched with 734 patients in the poor adherence group. After matching, the standardized differences were $<10 \%$, and $\mathrm{P}$ values were $>0.05$ for all variables, indicating that all baseline characteristics were well balanced between the two groups (Supplementary Tables S5 and S6).

High adherence was associated with lower relative risk of hospitalized AECOPD (OR=0.73, $\mathrm{p}=0.002$ ), lower hospitalized AECOPD rate (frequency) per patient-year $(R R=0.76 ; p=0.002)$, and lower hospitalized AECOPD treatment costs (1330 vs 1895 USD; $\mathrm{p}=0.012)$. Results were consistent with the multivariable regression results. 
Table I Baseline Characteristics of Patients in the Combination ICS/LABA Group

\begin{tabular}{|c|c|c|c|}
\hline \multirow[t]{2}{*}{ Characteristic } & \multirow{2}{*}{$\begin{array}{l}\text { High Adherence } \\
(n=903)\end{array}$} & \multirow{2}{*}{$\begin{array}{l}\text { Poor Adherence } \\
(n=2516)\end{array}$} & \multirow[t]{2}{*}{ P-value } \\
\hline & & & \\
\hline $\begin{array}{l}\text { Age (years), n (\%) } \\
<65 \\
65-74 \\
\geq 75 \\
\text { Missing }\end{array}$ & $\begin{array}{l}243(26.9) \\
346(38.3) \\
314(34.8) \\
0\end{array}$ & $\begin{array}{l}809(32.2) \\
825(32.8) \\
88 I(35.0) \\
I(0.0)\end{array}$ & 0.003 \\
\hline $\begin{array}{l}\text { Sex, n (\%) } \\
\text { Female } \\
\text { Male }\end{array}$ & $\begin{array}{l}139(15.4) \\
764(84.6)\end{array}$ & $\begin{array}{l}540(21.5) \\
1976(78.5)\end{array}$ & $<0.001$ \\
\hline $\begin{array}{l}\mathrm{CCl} \text { (No Age Adjustment) } \\
\text { Mean } \pm \text { SD } \\
\text { Median }\end{array}$ & $\begin{array}{l}0.03 \pm 0.18 \\
0\end{array}$ & $\begin{array}{l}0.02 \pm 0.18 \\
0\end{array}$ & 0.1307 \\
\hline $\begin{array}{l}\text { Pre-Index AECOPD Frequency, } \mathrm{n}(\%) \\
0 \\
\text { I outpatient/ED } \\
\geq 2 \text { outpatient/ED or having at least I hospitalized AECOPD }\end{array}$ & $\begin{array}{l}336(37.2) \\
|4|(15.6) \\
426(47.2)\end{array}$ & $\begin{array}{l}1,306(51.9) \\
285(11.3) \\
925(36.8)\end{array}$ & $<0.001$ \\
\hline $\begin{array}{l}\text { Pre-Index SABA Prescriptions, n (\%) } \\
0 \\
\mathrm{I}-3 \\
>3\end{array}$ & $\begin{array}{l}624(69.1) \\
209(23.2) \\
70(7.8)\end{array}$ & $\begin{array}{l}1831(72.8) \\
553(22.0) \\
132(5.3)\end{array}$ & 0.01 \\
\hline $\begin{array}{l}\text { Pre-Index LAMA Prescriptions, } n(\%) \\
0 \\
\mathrm{I}-3 \\
>3\end{array}$ & $\begin{array}{l}663(73.4) \\
84(9.3) \\
156(17.3)\end{array}$ & $\begin{array}{l}2049(81.4) \\
254(10.1) \\
213(8.5)\end{array}$ & $<0.001$ \\
\hline $\begin{array}{l}\text { Pre-Index Methylxanthines Prescriptions, n (\%) } \\
0 \\
1-3 \\
>3\end{array}$ & $\begin{array}{l}415(46.0) \\
196(21.7) \\
292(32.3)\end{array}$ & $\begin{array}{l}1540(61.2) \\
565(22.5) \\
411(16.3)\end{array}$ & $<0.001$ \\
\hline $\begin{array}{l}\text { Pre-Index COPD-Related Cost (USD) } \\
\text { Mean } \pm \text { SD } \\
\text { Median }\end{array}$ & $\begin{array}{l}1343 \pm 2832 \\
574\end{array}$ & $\begin{array}{l}992 \pm 2416 \\
224\end{array}$ & $<0.001$ \\
\hline $\begin{array}{l}\text { Pre-Index COPD Inpatient Visits, n (\%) } \\
0 \\
\mathrm{I} \\
\geq 2\end{array}$ & $\begin{array}{l}714(79.1) \\
113(12.5) \\
76(8.4)\end{array}$ & $\begin{array}{l}2,006(79.7) \\
302(12.0) \\
208(8.3)\end{array}$ & 0.906 \\
\hline
\end{tabular}

Note: All included patients used exclusively one category of drug on the index date.

Abbreviations: COPD, chronic obstructive pulmonary disease; ICS/LABA, inhaled corticosteroid/long-acting beta-2 agonist; AECOPD, acute exacerbation of chronic obstructive pulmonary disease; SD, standard deviation; SABA, short-acting beta-agonist; LAMA, long-acting muscarinic antagonist; $\mathrm{CCl}$, Charlson Co-morbidity Index; ED, emergency department; USD, United States dollar.

\section{Adjusted PDC Cutoff Point}

After adjusting the cutoff point of PDC to 0.5, among patients with COPD using the ICS/LABA combination maintenance therapy, 1626 patients were highly adherent, whereas the remaining 1793 were in the poor adherence group. High adherence still demonstrated better clinical and economic outcomes compared with the poor adherence group; however, between-group differences were smaller compared with the 0.8 PDC cutoff point. The relative risk of hospitalized AECOPD in the high adherence group was reduced by $12.9 \%$ (adjusted OR: 0.87 ; SE: $0.07 ; 95 \%$ CI: $0.74,1.03 ; \mathrm{p}=0.12$ ). The rate (frequency) of 
Table 2 Relative Risk of Hospitalized AECOPD (High Adherence Vs Poor Adherence) in COPD Patients During the Follow-Up Period

\begin{tabular}{|c|c|c|c|c|}
\hline & $\begin{array}{l}\text { Odds } \\
\text { Ratio }\end{array}$ & $\begin{array}{l}\text { Standard } \\
\text { Error }\end{array}$ & $95 \% \mathrm{Cl}$ & P-value \\
\hline $\begin{array}{l}\text { Combination ICS/ } \\
\text { LABA user }{ }^{\mathrm{a}}\end{array}$ & 0.65 & 0.06 & $0.54,0.79$ & $<0.001$ \\
\hline $\begin{array}{l}\text { Inhaled } \\
\text { bronchodilator user }\end{array}$ & 0.81 & 0.18 & $0.52,1.26$ & 0.339 \\
\hline Oral mucolytic user ${ }^{\mathrm{b}}$ & 2.51 & 0.55 & $1.63,3.87$ & $<0.001$ \\
\hline Test for interaction ${ }^{c, d}$ & - & - & - & 0.001 \\
\hline
\end{tabular}

Notes: 'Logistic regression model with the high adherence as the primary exposure and adjusted for age, gender, pre-index AECOPD frequency, Charlson comorbidity index, pre-index theophylline script number, pre-index SABA script number, pre-index LAMA script number, pre-index COPD related cost, and pre-index COPD inpatient visit times for inhaled bronchodilator and ICS/LABA user. bLogistic regression model with the high adherence as the primary exposure and adjusted for age, gender, pre-index AECOPD frequency, Charlson comorbidity index, pre-index $L A B A$ script number, pre-index SABA script number, pre-index LAMA script number, pre-index COPD related cost, and pre-index COPD inpatient visit times for oral mucolytic user. 'Logistic regression model with the high adherence as the primary exposure and adjusted for age, gender, pre-index AECOPD frequency, Charlson comorbidity index, pre-index SABA script number, pre-index LAMA script number, pre-index COPD related cost, and pre-index COPD inpatient visit times for the model with interaction. ${ }^{\mathrm{d}}$ Test for interaction between drug category and adherence status (high or poor).

Abbreviations: COPD, chronic obstructive pulmonary disease; AECOPD, acute exacerbation of chronic obstructive pulmonary disease; $\mathrm{Cl}$, confidence interval; ICS/LABA, inhaled corticosteroid/long-acting beta-2 agonist; SABA, short-acting beta-agonist; LAMA, long-acting muscarinic antagonist.

hospitalized AECOPD per patient-year was reduced by 11.3\% (RR: 0.89; SE: 0.05; 95\% CI: 0.79, 1.00; $\mathrm{p}=0.053$ ). Hospitalized AECOPD cost was reduced by $33.3 \%$. Predicted mean cost was 1624 vs 2434 USD, adjusted mean difference (SE) was -810 USD (327) (95\% CI: $-1,451,-168 ; \mathrm{p}<0.001)$.

\section{Discussion}

To the best of our knowledge, the present study is the first to examine the association between adherence to maintenance medication and clinical and economic outcomes and to explore the interaction effect of drug category among patients with COPD in a real-world setting in China. Among the three maintenance groups, only high adherence to the ICS/LABA combination was significantly associated with reduced risk, lower frequency, and cost savings for hospitalized AECOPD. Our study complements randomized, controlled clinical trials by including a heterogeneous patient population with different severity levels and reflects realworld adherence to COPD maintenance medications without protocol-driven interventions. The strength of the study was that, although this study was limited to one city, given universal medical insurance coverage, these findings have implications for COPD care more broadly in China. ${ }^{14}$

Among the three categories of maintenance therapies, the ICS/LABA combination has been proven to alleviate symptoms and decrease the risk of exacerbation of COPD. ${ }^{15}$ The GOLD 2019 guidelines state that

an ICS combined with a LABA is more effective than the individual components in improving lung function and health status and reducing exacerbations in patients with exacerbations and moderate-to-very severe COPD. (Evidence A)

1 Study results from a randomized clinical trial run in a primary healthcare setting in the United Kingdom comparing the ICS/LABA combination with usual care showed an $8.4 \%$ reduction of exacerbations (moderate-tosevere level). ${ }^{16}$ With regards to oral therapy, meta-analysis

Table 3 Incident Rate Ratio of Hospitalized AECOPD Visits (High Adherence Vs Poor Adherence) Among COPD Patients During the Follow-Up Period

\begin{tabular}{|l|l|l|l|l|l|l|l|l|}
\hline \multirow{2}{*}{} & \multicolumn{2}{l}{ Incident Rate Ratio } & \multicolumn{2}{l|}{ High Adherence } & \multicolumn{2}{l|}{ Poor Adherence } \\
\cline { 2 - 9 } & Mean & SE & $\mathbf{9 5 \%} \mathbf{~ C l}$ & P-value & Predicted Mean & SE & Predicted Mean & SE \\
\hline Combination ICS/LABA user $^{\mathrm{a}}$ & 0.76 & 0.06 & $0.65,0.87$ & $<0.001$ & 0.55 & 0.04 & 0.73 & \\
Inhaled bronchodilator user $^{\mathrm{a}}$ & 0.83 & 0.14 & $0.59,1.17$ & 0.28 & 0.49 & 0.06 & 0.59 & 0.03 \\
Oral mucolytic user $^{\text {b }}$ & 1.82 & 0.21 & $1.45,2.29$ & $<0.001$ & 1.69 & 0.19 & 0.92 & 0.06 \\
Test for interaction $^{\text {c,d }}$ & - & - & - & $<0.001$ & - & - & - & 0.02 \\
\hline
\end{tabular}

Notes: ${ }^{a}$ Negative binomial regression model with the high adherence as the primary exposure and adjusted for age, gender, pre-index AECOPD frequency, Charlson comorbidity index, pre-index theophylline script number, pre-index SABA script number, pre-index LAMA script number, pre-index COPD related cost, and pre-index

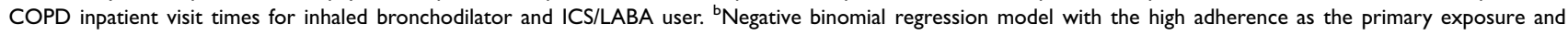
adjusted for age, gender, pre-index AECOPD frequency, Charlson comorbidity index, pre-index LABA script number, pre-index SABA script number, pre-index LAMA script number, pre-index COPD related cost, and pre-index COPD inpatient visit times for oral mucolytic user. ${ }^{c}$ Negative binomial regression model with the high adherence as the primary exposure and adjusted for age, gender, pre-index AECOPD frequency, Charlson comorbidity index, pre-index SABA script number, pre-index LAMA script number, pre-index COPD related cost, and pre-index COPD inpatient visit times for the model with interaction. ${ }^{\mathrm{d}}$ Test for interaction between drug category and adherence status (high or poor).

Abbreviations: COPD, chronic obstructive pulmonary disease; AECOPD, acute exacerbation of chronic obstructive pulmonary disease; SE, standard error; Cl, confidence interval; ICS/LABA, inhaled corticosteroid/long-acting beta-2 agonist; SABA, short-acting beta-agonist; LAMA, long-acting muscarinic antagonist. 
Table 4 Hospitalized AECOPD Cost Comparison (USD) Between High Adherence Cohort and Poor Adherence Cohort Among COPD Patients During the Follow-Up Period

\begin{tabular}{|c|c|c|c|c|c|c|c|c|}
\hline & \multicolumn{2}{|l|}{ High Adherence } & \multicolumn{2}{|l|}{ Poor Adherence } & \multicolumn{4}{|c|}{ Mean Difference } \\
\hline & Predicted Mean & SE & Predicted Mean & SE & Mean & SE & $95 \% \mathrm{Cl}$ & P-value \\
\hline Combination ICS/LABA user ${ }^{a}$ & 1393 & 186 & 2242 & 235 & -848 & 299 & $-1435,-262$ & $<0.001$ \\
\hline Inhaled bronchodilator user ${ }^{\mathrm{a}}$ & 1556 & 416 & |87| & 480 & -315 & 636 & $-\mid 560,931$ & 0.46 \\
\hline Oral mucolytic user ${ }^{\mathrm{b}}$ & 5273 & $120 \mid$ & 2053 & 85 & 3221 & 1204 & 860,5581 & $<0.001$ \\
\hline Test for interaction ${ }^{c, d}$ & - & - & - & - & - & - & - & $<0.001$ \\
\hline
\end{tabular}

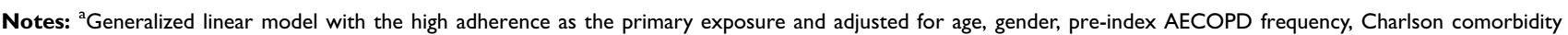
index, pre-index theophylline script number, pre-index SABA script number, pre-index LAMA script number, pre-index COPD related cost, pre-index COPD inpatient visit times for inhaled bronchodilator and ICS/LABA user. ${ }^{b}$ Generalized linear model with the high adherence as the primary exposure and adjusted for age, gender, pre-index AECOPD frequency, Charlson comorbidity index, pre-index LABA script number, pre-index SABA script number, pre-index LAMA script number, pre-index COPD related cost, pre-index COPD inpatient visit times for oral mucolytic user. ${ }^{\circ}$ Generalized linear model with the high adherence as the primary exposure and adjusted for age, gender, pre-index AECOPD frequency, Charlson comorbidity index, pre-index SABA script number, pre-index LAMA script number, pre-index COPD related cost, pre-index COPD inpatient visit times for the model with interaction. ${ }^{d}$ Test for interaction between drug category and adherence status (high or poor).

Abbreviations: COPD, chronic obstructive pulmonary disease; AECOPD, acute exacerbation of chronic obstructive pulmonary disease; SE, standard error; CI, confidence interval; USD, United States dollar; ICS/LABA, inhaled corticosteroid/long-acting beta-2 agonist; SABA, short-acting beta-agonist; LAMA, long-acting muscarinic antagonist.

results provided evidence that although long-term highdose N-acetylcysteine (NAC) treatment might lead to a lower rate of COPD exacerbations, ${ }^{17}$ low-dose NAC was insufficient in providing benefit to COPD patients. ${ }^{18}$ Actually, the BRONCUS trial showed that NAC was ineffective in preventing exacerbations in patients with COPD. ${ }^{19}$ Schermer et al. ${ }^{20}$ even found that 3 years' treatment with low-dose NAC increased the exacerbation rate. The result of the present study was that patients taking oral mucolytics and having high adherence had worse AECOPD outcomes than patients with poor adherence. One possible reason for high adherence having worse impact on AECOPD in the oral mucolytic group in our study could be the low daily dosage, yet dosage information was unavailable in the claims database. Therefore, additional study is necessary to determine the exact causes.

In fact, in China, we seek to apply the current GOLD guidelines, as in other countries. Differences with other countries might lie in the health care system and the insurance system in China. The escalating costs of health care with the health care reform were a cause of transient poverty for many families. Hence, social health insurance systems were introduced gradually, and, currently, about $95 \%$ of the population is covered by medical insurance. ${ }^{21}$ Despite this good coverage, the results showed that COPD maintenance treatment in China was not optimal. According to the GOLD guidelines, bronchodilators, including long-acting beta-agonists and anticholinergics (alone or in combination), should be the basic pharmacological therapy for all patients with COPD. ${ }^{1}$ However, our study found that among the 11,708 patients with COPD identified for the analysis, $65.5 \%$ used oral medications only on the index date, among which only $25.9 \%$ had inhaled bronchodilator or ICS/LABA combination prescriptions during the follow-up period. One potential reason could be the lack of knowledge on standardized treatment of COPD. ${ }^{3}$ He et al carried out a study evaluating real-world pharmacologic therapy usage for COPD via face-to-face interviews of 723 patients with stable disease and 258 pulmonary physicians in six cities in China. The results indicated that mucolytics were the most commonly prescribed medications. ${ }^{22}$ Physicians' lack of knowledge of the GOLD guidelines could explain the disproportionate use of oral medications for COPD treatment in the present study; indeed, a survey of primary care providers indicated only $20 \%$ were fully knowledgeable about standardized COPD pharmacologic therapy. ${ }^{22}$ Besides, the cost might be another driving factor.

Our study also showed that adherence to maintenance therapy was poor among Chinese patients with COPD. For inhaled bronchodilator users, only $36.3 \%$ of patients were deemed to be highly adherent to treatment, and for the ICS/LABA combination, adherence to treatment was even lower $(29.2 \%)$. For patients taking oral medications, only $1.7 \%$ was considered highly adherent.

The situation of unideal COPD maintenance treatment is not alone in China. A study based on insurance claims in the USA showed that patients who started long-term bronchodilator monotherapy showed high rates of treatment discontinuation or augmentation, as well as high rates of use of rescue therapy, and high treatment costs. ${ }^{23}$ Another realworld study from the USA showed that patients starting a LAMA/LABA combination had higher adherence than 
patients starting an ICS/LABA combination. ${ }^{24}$ Two studies from the United Kingdom revealed that the rate of prescribing according to the guidelines was low and that many patients were over-treated, and with poor adherence to treatment. ${ }^{25,26}$ Therefore, the poor adherence and guideline compliance observed in China is probably not different from that observed in other countries.

Similar to our findings, previous research showed that poor adherence to maintenance treatment was associated with unfavorable clinical and economic outcomes. A large retrospective study using a claims database showed that higher adherence rates to COPD maintenance medication were correlated with reduced emergency care usage (annual number of inpatient days, inpatient visits and emergency room visits) and lower healthcare spending in patients with COPD. ${ }^{13}$ Vestbo et al conducted a post hoc analysis of the multinational TORCH trial, where 6112 patients with COPD were followed up for 3 years. There was a significant association between good adherence and lower exacerbations. ${ }^{27}$

Our study has some limitations. First, administrative claims data do not include lung function data. ${ }^{15}$ This limitation was partially addressed by controlling for baseline COPD severity in terms of COPD treatment expenditure, AECOPD frequency (rate), and COPD related hospitalization frequency (rate). These surrogates for COPD severity have been used in previous COPD studies using claims data. ${ }^{15,28}$ Second, our measurement of adherence was based on prescription filled; the claims database cannot account for whether the medications were actually taken as prescribed. Mishandling or misuse of inhalers is associated with reduced disease control, ${ }^{13}$ but there is no evidence that these factors correlated with adherence status, so they would not distort the association between adherence and outcomes. Third, patients were grouped as "long-term bronchodilators", "fixed combinations of inhaled corticosteroids and long-acting beta-agonists" and "oral medications such as methylxanthine or mucolytic agents" based on the treatment on the index date. The potential pitfall of such "intention-to-treat" study design was that patient might switch to other maintenance therapies or discontinued treatment during follow-up period, which would potentially dilute the association to be explored. Even under this context, the results still demonstrated significant association between adherence and outcomes in the ICS/LABA combination user group. Finally, given the small sample size for the inhaled bronchodilator group, we might lack the power to detect a difference between the high and poor adherence groups.

\section{Conclusions}

High adherence to the ICS/LABA combination maintenance therapy was associated with improved clinical and economic outcomes in a cohort of Chinese patients with COPD. The results also highlighted the need to improve adherence to such therapy in this setting.

\section{Abbreviations}

AECOPD, acute exacerbations of chronic obstructive pulmonary disease; COPD, chronic obstructive pulmonary disease; ICS/LABA, inhaled corticosteroid/long-acting beta-2 agonist; DOT, duration of therapy; ED, emergency department; MD, mean difference; OCS, oral corticosteroid; OR, odds ratio; PDC, proportion of days covered; RR, relative risk; SE, standard error; YLLs, years of life lost.

\section{Data Sharing Statement}

The datasets used and/or analyzed during the current study are available from the corresponding author on reasonable request.

\section{Ethics and Consent Statement}

Because only de-identified records were used in the analysis, ethical approval and informed consent were not required consistent with the local medical research policy using electronic health data.

\section{Acknowledgments}

The authors would like to thank Shanghai MedSci Healthcare for providing medical writing support, funded by AstraZeneca, China, in accordance with the Good Publication Practice (GPP3) guidelines.

\section{Author Contributions}

All authors made substantial contributions to conception and design, acquisition of data, or analysis and interpretation of data; took part in drafting the article or revising it critically for important intellectual content; gave final approval of the version to be published; and agree to be accountable for all aspects of the work.

\section{Funding}

This study was supported by AstraZeneca, China.

\section{Disclosure}

He Wang and Hongyan Shang are employees of AstraZeneca, China. Yue Gao reports grants from AstraZeneca Investment 
(China) Co. Ltd., during the conduct of the study; grants from AstraZeneca Investment (China) Co. Ltd., outside the submitted work. The authors declare no other conflicts of interest in this work.

\section{References}

1. Vogelmeier CF, Criner GJ, Martinez FJ, et al. Global strategy for the diagnosis, management and prevention of chronic obstructive lung disease 2017 report. Respirology. 2017;22:575-601.

2. World Health Organization. The global burden of disease, 2004 update. http://www.who.int/healthinfo/global_burden_disease/GBD report_2004update_full.pdf?ua $=1$.

3. Fang X, Wang X, Bai C. COPD in China: the burden and importance of proper management. Chest. 2011;139:920-929. doi:10.1378/ chest.10-1393

4. G. B D. Causes of death collaborators. global, regional, and national age-sex specific mortality for 264 causes of death, 1980-2016: a systematic analysis for the global burden of disease study 2016. Lancet. 2017;390:1151-1210. doi:10.1016/S01406736(17)32152-9

5. Guarascio AJ, Ray SM, Finch CK, Self TH. The clinical and economic burden of chronic obstructive pulmonary disease in the USA. CEOR. 2013;5:235-245. doi:10.2147/CEOR.S34321

6. Fang L, Gao P, Bao H, et al. Chronic obstructive pulmonary disease in China: a nationwide prevalence study. Lancet Res Med 2018;6:421-430. doi:10.1016/S2213-2600(18)30103-6

7. Yang G, Wang Y, Zeng Y, et al. Rapid health transition in China, 1990-2010: findings from the global burden of disease Study 2010 Lancet. 2013;381:1987-2015. doi:10.1016/S0140-6736(13)61097-1

8. Tan WC, Ng TP. COPD in Asia: where east meets west. Chest. 2008;133:517-527. doi:10.1378/chest.07-1131

9. He QY, Zhou X, Xie CM, Liang ZA, Chen P, Wu CG. [Impact of chronic obstructive pulmonary disease on quality of life and economic burden in Chinese urban areas]. Zhonghua Jie He He Hu Xi Za Zhi. 2009;32:253-257.

10. Restrepo RD, Alvarez MT, Wittnebel LD, et al. Medication adherence issues in patients treated for COPD. Int $J$ Chron Obstruct Pulmon Dis. 2008;3:371-384. doi:10.2147/COPD.S3036

11. Stuart BC, Simoni-Wastila L, Zuckerman IH, et al. Impact of maintenance therapy on hospitalization and expenditures for medicare beneficiaries with chronic obstructive pulmonary disease. $\mathrm{Am}$ $J$ Geriatr Pharmacother. 2010;8:441-453. doi:10.1016/j.amjopharm. 2010.10.002

12. Sanduzzi A, Balbo P, Candoli P, et al. COPD: adherence to therapy. Multidiscip Respir Med. 2014;9:60. doi:10.1186/2049-6958-9-60

13. van Boven JF, Chavannes NH, van der Molen T, Rutten-van Molken MP, Postma MJ, Vegter S. Clinical and economic impact of non-adherence in COPD: a systematic review. Respir Med. 2014;108:103-113. doi:10.1016/j.rmed.2013.08.044

14. Yu H. Universal health insurance coverage for 1.3 billion people: what accounts for China's success? Health Policy. 2015;119:1145-1152. doi:10.1016/j.healthpol.2015.07.008
15. Simoni-Wastila L, Wei YJ, Qian J, et al. Association of chronic obstructive pulmonary disease maintenance medication adherence with all-cause hospitalization and spending in a medicare population. Am J Geriatr Pharmacother. 2012;10:201-210. doi:10.1016/j.amjopharm.2012.04.002

16. Vestbo J, Leather D, Diar Bakerly N, et al. Effectiveness of fluticasone furoate-vilanterol for COPD in clinical practice. $N$ Engl J Med. 2016;375:1253-1260. doi:10.1056/NEJMoa1608033

17. Shen Y, Cai W, Lei S, Zhang Z. Effect of high/low dose N-acetylcysteine on chronic obstructive pulmonary disease: a systematic review and meta-analysis. Copd. 2014;11:351-358. doi:10.3109/15412555.2013. 858315

18. Cazzola M, Calzetta L, Page C, et al. Influence of N-acetylcysteine on chronic bronchitis or COPD exacerbations: a meta-analysis. Eur Res. 2015;24:451-461. doi:10.1183/16000617.00002215

19. Decramer M, Rutten-van Molken M, Dekhuijzen PN, et al. Effects of $\mathrm{N}$-acetylcysteine on outcomes in chronic obstructive pulmonary disease (bronchitis randomized on NAC cost-utility study, BRONCUS): a randomised placebo-controlled trial. Lancet. 2005;365:1552-1560. doi:10.1016/S0140-6736(05)66456-2

20. Schermer T, Chavannes N, Dekhuijzen R, et al. Fluticasone and $\mathrm{N}$-acetylcysteine in primary care patients with COPD or chronic bronchitis. Respir Med. 2009;103:542-551. doi:10.1016/j.rmed. 2008.11.003

21. Miao Y, Gu J, Zhang L, He R, Sandeep S, Wu J. Improving the performance of social health insurance system through increasing outpatient expenditure reimbursement ratio: a quasi-experimental evaluation study from rural China. Int J Equity Health. 2018;17:89. doi:10.1186/s12939-018-0799-8

22. Zhang R, He Q. Awareness of knowledge of COPD by doctors in district and community hospitals. Chin J Prev Control Chronic Dis. 2009; 17:61-63.

23. Bengtson LGS, DePietro M, McPheeters J, Fox KM. Real-world outcomes in patients with chronic obstructive pulmonary disease initiating long-acting mono bronchodilator therapy. Ther Adv Respir Dis. 2018;12:1753466618772750. doi:10.1177/1753466618772750

24. Moretz C, Sharpsten L, Bengtson LG, et al. Real-world effectiveness of umeclidinium/vilanterol versus fluticasone propionate/salmeterol as initial maintenance therapy for chronic obstructive pulmonary disease (COPD): a retrospective cohort study. Int J Chron Obstruct Pulmon Dis. 2019;14:1721-1737. doi:10.2147/COPD.S204649

25. Halpin DMG, de Jong HJI, Carter V, Skinner D, Price D. Distribution, temporal stability and appropriateness of therapy of patients with COPD in the UK in relation to GOLD 2019. E Clin Med. 2019;14:32-41. doi:10.1016/j.eclinm.2019.07.003

26. Gruffydd-Jones K, Brusselle G, Jones R, et al. Changes in initial COPD treatment choice over time and factors influencing prescribing decisions in UK primary care: in UK primary care: a real-world, retrospective, observational. NPJ Prim Care Res Med. 2016;26:16002. doi:10.1038/npjpcrm.2016.2

27. Vestbo J, Anderson JA, Calverley PM, et al. Adherence to inhaled therapy, mortality and hospital admission in COPD. Thorax. 2009;64:939-943. doi:10.1136/thx.2009.113662

28. Toy EL, Beaulieu NU, McHale JM, et al. Treatment of COPD: relationships between daily dosing frequency, adherence, resource use, and costs. Respir Med. 2011;105:435-441. doi:10.1016/j.rmed.2010.09.006

\section{Publish your work in this journal}

The International Journal of COPD is an international, peer-reviewed journal of therapeutics and pharmacology focusing on concise rapid reporting of clinical studies and reviews in COPD. Special focus is given to the pathophysiological processes underlying the disease, intervention programs, patient focused education, and self management protocols. This journal is indexed on PubMed Central, MedLine and CAS. The manuscript management system is completely online and includes a very quick and fair peer-review system, which is all easy to use. Visit http://www.dovepress.com/testimonials.php to read real quotes from published authors. 